\title{
A Narrative Review of the Risk Factors for Cancer and the Preventive Opportunities: Current Status, Future Perspectives, and Implications for India
}

\author{
Vinod K. Ramani ${ }^{1,2} \quad$ Ganesha D.V. ${ }^{3} \quad$ Radheshyam Naik ${ }^{4}$ \\ ${ }^{1}$ Department of Preventive Oncology, Healthcare Global Enterprise \\ Ltd., Bangalore, Karnataka, India \\ ${ }^{2}$ Department of Public health, Ramaiah University of Applied \\ Sciences, Bangalore, Karnataka, India \\ ${ }^{3}$ Department of Medical Oncology, St. John's Medical College and \\ Hospital, Bangalore, Karnataka, India \\ ${ }^{4}$ Department of Medical Oncology, Healthcare Global Enterprise \\ Ltd., Bangalore, India
}

\begin{abstract}
Address for correspondence Vinod K. Ramani, MBBS, MD, MPH, Department of Preventive Oncology, Healthcare Global Enterprise Ltd., K.R. Road, Bangalore 560027, Karnataka, India (e-mail: drvinod.r@hcgel).
\end{abstract}

Asian J Oncol 2021;8:47-56.

\begin{abstract}
Keywords

- preventive health services

- neoplasms by sites

- early detection of cancer

- exercise

- dietary habits
\end{abstract}

Introduction Clinical cancer can arise from heterogenous pathways through various genetic mutations. Although we cannot predict the timeline by which an individual will develop cancer, certain risk assessment tools can be used among high-risk groups for focusing the preventive activities. As primary level of cancer prevention, healthy lifestyle approach is being promoted. The etiological factors for lung cancer include by-products of industrialization and air pollution. We need to factor the increase in household air pollution as well.

Methods "PubMed" database and Google search engines were used for searching the relevant articles. Search terms with Boolean operators used include "Cancer prevention," "Missed opportunities in cancer causation," and "incidence of risk factors." This review includes 20 studies and other relevant literature that address the opportunities for cancer prevention.

Body The narrative describes the association between many of the risk factors and development of cancer. This includes tobacco, alcohol, infections, air pollution, physical inactivity, diet, obesity, screening and preventive strategies, chemoprevention, biomarkers of carcinogenesis, and factors that prolong the diagnosis of cancer.

Discussion Reports from basic science research provide evidence on the potential of biologically active food components and pharmacological agents for mitigating the risk of cancer and its progression. However, some reports from observational studies and randomized trials have been inconsistent. We need to recognize the impact of sociodemographic factors such as age, sex, ethnicity, culture, and comorbid illness on preventive interventions. Spiral computed tomographic scan is a robust tool for early detection of lung cancer.

Conclusion Infectious etiology for specific cancers provides opportunities for prevention and treatment. The complex interplay between man and microbial flora needs to be dissected, for understanding the pathogenesis of relevant malignancies. For reducing the morbidity of cancer, we need to focus on prevention as a priority strategy and intervene early during the carcinogenic process. published online

June 15, 2021
DOI https://doi.org/

$10.1055 / \mathrm{s}-0041-1731092$

ISSN 2454-6798 (c) 2021. Spring Hope Cancer Foundation \& Young Oncologist Group of Asia.

This is an open access article published by Thieme under the terms of the Creative Commons Attribution-NonDerivative-NonCommercial-License, permitting copying and reproduction so long as the original work is given appropriate credit. Contents may not be used for commercial purposes, or adapted, remixed, transformed or built upon. (https://creativecommons.org/licenses/by-nc-nd/4.0/).

Thieme Medical and Scientific Publishers Pvt. Ltd. A-12, 2nd Floor, Sector 2, Noida-201301 UP, India 


\section{Introduction}

Globally, noncommunicable diseases (NCDs) account for $60 \%$ of all the deaths. In India, NCDs account for $50 \%$ of all deaths and cancer accounts for one-fifth of all NCDs. ${ }^{1}$ Among the cancer morbidity in India, 70\% are preventable through modification of the risk factors. This includes $40 \%$ due to tobacco, $20 \%$ due to infection, and $10 \%$ related to others. ${ }^{1}$ Considering a median life expectancy of 74 years, one in eight men and one in nine women are expected to suffer from cancer. ${ }^{1}$ Infectious agents tend to cause $20 \%$ of human cancers and they rank second to tobacco as potentially preventable causes in humans. ${ }^{2}$

An estimated 12 to $20 \%$ of cancers worldwide are due to human tumor viruses. These viruses when compared with others are unusual, because they infect the host cells but do not kill them. Thus, such viruses tend to establish persistent infections. Epstein-Barr virus (EBV) is associated with Burkitt's lymphoma (BL), nasopharyngeal carcinoma, and some forms of Hodgkin's disease. The virus may remain latent in the nasopharyngeal epithelial cells and smoking could possibly reactivate EBV causing nasopharyngeal carcinoma.

Lyratzopoulos et $\mathrm{al}^{3}$ conducted a retrospective case review of cancer cohorts for deriving evidence regarding the missed opportunities for cancer diagnosis. The authors categorize the same in three main phases:

1. The diagnostic assessment by the physician may result in a safety net/wait and see reasoning and inappropriate interpretation.

2. Missing out on multiple investigations (often at different times and location) and their associated actions.

3. The patient may be lost to follow-up or the health system may be unable to "close the loop" due to inadequate diagnostic follow-up and coordination.

With increase in the level of particulate matter (PM) 2.5 in air either due to agricultural or vehicular emission, there exists a concomitant risk of developing lung cancer (group 1,2A). Household combustion of coal causes indoor air pollution that is carcinogenic to humans (group 1). Some of the potentially modifiable risk factors include tobacco and/or alcohol consumption, infections, and dietary factors. Country and geography specific variations are present for these preventable cancers.

When compared with other malignancies such as breast, prostate, and colon, patients with lung cancer often present at an advanced stage where surgical intervention may not be feasible. Few studies report that lung cancer screening using low-dose computed tomography (CT) scans has been effective in reducing mortality by $20 \%{ }^{4}$ Certain population at high risk for lung cancer, who are defined by their smoking history, previous history of tobacco-induced cancer, family history, and altered pulmonary function should be subjected to such screening interventions.

\section{Methods}

"PubMed" database and Google search engines were used for searching the relevant articles. Search terms with Boolean operators used include "Cancer prevention," "Missed opportunities in cancer causation," and "incidence of risk factors." The results of this search yield research articles that contextually provide details of the relevant concepts. The criterion used for reviewing these articles includes their relevance to the defined review question, which are the missed opportunities for cancer prevention and the relevant screening and diagnostic interventions therewith. These studies were reviewed for reference to screening process, laboratory investigations, early diagnosis of cancer symptoms, issues influencing delivery of healthcare systems such as referral pathways, therapeutic regimen, and other relevant concepts. This review includes 20 studies and other relevant literature that address the opportunities for cancer prevention.

\section{Body}

Carcinogenesis involves a step-wise accumulation process of genetic and epigenetic changes, which eventually result in a cell of malignant phenotype. This process involves exposure to carcinogen, formation of DNA adducts, inflammatory changes, oxidative stress, mutation, and epigenetic alterations that cause the characteristic pathologic changes in cancer. These pathologic changes include signaling for sustained proliferative growth, evasion of growth suppression, resistance to cell death, immortality of replication, angiogenesis, invasion, metabolism, evasion of host immunity, and reprogramming of energy metabolism. Such changes are a reflection of genomic instability.

- Table 1 reproduced from Nair et al ${ }^{4}$ depicts the common risk factors for cancer and their contribution to cancer mortality. This narrative describes the association between many of these risk factors and the risk of cancer development.

\section{Tobacco}

Intake of tobacco (both smoke and smokeless forms) accounts for $21 \%$ of total cancer deaths worldwide. ${ }^{1}$ It increases the risk of lung cancer as well as 15 other cancers. Studies ${ }^{6}$ also demonstrate an increased risk following prolonged exposure to environmental tobacco smoke (second-hand aerosol from tobacco). Beedi smoking that is more prevalent in India is

Table 1 Risk factors for cancer

\begin{tabular}{|l|l|l|}
\hline S. No. & Risk factor & $\begin{array}{l}\text { \% of cancer deaths } \\
\text { in 35-64 years age } \\
\text { group (estimate) }\end{array}$ \\
\hline 1 & Tobacco & $30-40$ \\
\hline 2 & Alcohol & $3-10$ \\
\hline 3 & Diet & Not known \\
\hline 4 & $\begin{array}{l}\text { Reproductive and sexual } \\
\text { behavior }\end{array}$ & 10 \\
\hline 5 & Occupation & $6-8$ \\
\hline 6 & Pollution & 2 \\
\hline 7 & Industrial products & 1 \\
\hline 8 & $\begin{array}{l}\text { Medicines and medical } \\
\text { procedures }\end{array}$ & 1 \\
\hline 9 & Geophysical factors & 3 \\
\hline
\end{tabular}


known to be more carcinogenic than the cigarettes. The benefits of tobacco cessation are applicable not just for cancer prevention but also for reducing the incidence of cardiovascular and pulmonary diseases. Its cessation depends on the interplay of sociocultural habits, personal awareness, and government legislation. Personal intervention may require behavioral counseling or pharmacotherapy or both. Evidence shows that those who initiate smoking at a young age tend to smoke for longer years, and the risk of lung cancer has a strong association with the duration of smoking than daily consumption. ${ }^{7}$

Passive smokers are exposed to both the mainstream and side stream smoke. During 2004, globally $40 \%$ of children, $33 \%$ of male nonsmokers, and $35 \%$ of female nonsmokers were exposed to second-hand smoke that resulted in $1 \%$ of worldwide mortality. ${ }^{7}$ The confounding effect of tea consumption along with smoking has been reported in Mu et al's ${ }^{7}$ study. In this study, tea drinking was found to have protective effect with an adjusted odds ratio (OR) of 0.5 in nonsmokers and 0.45 in smokers. The 5 " $\mathrm{A}$ " method is useful for individuals who seek to quit (Ask, Advise, Assess, Assist and Arrange), and the 5 "R" method for those who are not yet willing to quit (Relevance of quitting, Risk of continuing tobacco, Rewards of quitting, Roadblocks to quitting and Repeat these at each visit). Subsequent to cessation of smoking, there is a significant residual risk of developing lung cancer.

Benzo(a)pyrene, a carcinogen found in cigarette smoke, is metabolically activated by the P450 family (CYP1A1) of hepatic enzymes. Such chemically active intermediate metabolites can bind to DNA and cause gene dysfunction. These products are detoxified by Glutathione-S-transferase, $\mathrm{N}$-acetyl transferase, and epoxide hydrolase. Modified metabolic activity is a result of polymorphisms and/or gene deletions. Polymorphisms can also occur in enzymes responsible for DNA repair. Genetic alterations among these enzymes can have effects on the individual's risk of developing lung cancer. ${ }^{8}$

Among smokers, relevant genetic abnormalities could be found among histologically normal bronchial epithelial cells. Thus, we could infer that a stepwise accumulation of genetic abnormalities leads to the development of cancer. Beyond the level of histological dysplasia, assessment of the accumulation of genetic alterations also provides information regarding the prognosis of lung cancer. The same could be done by fluorescence in situ hybridization, polymerase chain reaction-based loss of heterozygosity, comparative genomic hybridization, or sequence analysis. ${ }^{4}$ An early event in the development of squamous cell lung cancer (SCLC) includes SOX2 gene amplification (before other genetic changes) that is encoded on the chromosome 3q26. In some cases, specific epidermal growth factor receptor (EGFR), HER2 (human epidermal growth factor receptor 2), EML4-ALK (echinoderm microtubule associated protein like 4-anaplastic lymphoma kinase gene), or KRAS (Kirsten rat sarcoma viral oncogene homolog) mutations could precede the development of lung cancer. ${ }^{9}$

Spiral CT scanning provides a tool for early detection of lung cancer, as a complete image of the thorax can be obtained during a single hold of breath. ${ }^{10}$ This can locate considerably smaller cancers more frequently than with previous tools used for detection of lung cancer. Chest radiography is known to advance the diagnosis from clinical detection by $\sim 1$ year. ${ }^{10}$ As computer-assisted diagnosis continues to evolve, it needs to be integrated into routine cancer screening and clinical management.

\section{Alcohol}

Alcohol consumption contributes to $4 \%$ of all cancers worldwide. ${ }^{1}$ Alcohol is causally associated with cancers of the oropharynx and larynx, esophagus, breast, liver, and colon. For all these cancer sites, there is a dose-response relationship with no apparent threshold: the higher the average level of consumption, the higher the risk of cancer incidence. ${ }^{11}$ The dynamics of alcohol consumption are complicated due to peer pressure and social cultures. It is identified as a chronic disease with genetic, psychosocial, and environmental factors. Ethanol (pure alcohol) is a procarcinogen found in all alcoholic beverages. ${ }^{11}$ International Agency for Research on Cancer (IARC) labels procarcinogens as substances that transform into carcinogens by their metabolism. ${ }^{11}$ World Cancer Research Fund/American Institute for Cancer Research recommends that for prevention of cancer, it is best to avoid consumption of alcohol. ${ }^{12}$ We need to delineate how active alcohol use affects cancer treatment, the risk of recurrence, and overall prognosis, its interaction with oral chemotherapy and supportive care medications. Potential strategies to decrease the burden of cancer include initiatives for preventing consumption of alcohol.

The risk of cancer after alcohol cessation may be higher than that observed for current consumers of alcohol, because former drinkers might have been exposed to high doses of alcohol exposure. We need to design prospective studies that could longitudinally quantify the amount of alcohol consumed and the duration of cessation, which will characterize the impact of alcohol cessation on the risk of cancer. A synergistic interaction also exists between alcohol consumption and cigarette smoking, the biological underpinning of which may, however, not yet be explained. Genetic predisposition may amplify the toxic and mutagenic effects of alcohol consumption. Alcohol consumers with the inactive form of aldehyde dehydrogenase-2 experience excessive accumulation of acetaldehyde, which increases the susceptibility to alcohol induced cancer. Other mechanisms include oxidative stress, change in level of sex hormones, folate metabolism, DNA methylation, and cirrhosis leading to cancer. ${ }^{12}$ Continuing the habit after cancer diagnosis could be associated with risk of its recurrence and/or secondary tumor.

Alcohol abuse induced comorbidities and subclinical factors such as nutritional deficiencies, immunosuppression, and cardiovascular insufficiencies could complicate the choice of treatment in cancer patients. We need to establish the impact of awareness initiatives on alcohol use in the community. Given the dose-response relationship between the level of alcohol use and cancer, policy initiatives should include population level measures such as higher taxation of alcoholic beverages, restrictions on availability, and ban on marketing. ${ }^{11}$ 


\section{Infections}

Infections account for 20 to $25 \%$ of cancer cases worldwide $^{1}$ and are known etiological agents for cancers of stomach, cervix, and liver. Its prevalence is high in the developing Countries, due to the limited infection-prevention practices. Common infections include viruses such as human papilloma virus (HPV), hepatitis B and C virus (HBV and HCV), human T-cell lymphotropic virus (HTLV-1), human immunodeficiency virus (HIV), human herpes virus 8 and EBV. However, nonviral infections implicated in cancer causation include Helicobacter pylori (H. pylori) and some helminths (Clonorchis sinensis, Schistosoma haematobium).

HPV $16 / 18$ has been detected in 80 to $85 \%$ of cervical cancers $^{12}$ however, few Indian women are identified to be at risk. HPV-16 is found in $90 \%$ of the HPV-positive oropharyngeal cancers (OPC). HPV-positive OPCs usually develop at a younger age and are less often associated with cigarette smoking than are the HPV-negative cancers. ${ }^{2}$ India has 34 million carriers of HBV, and the risk of hepatocellular carcinoma in carriers of HBV and $\mathrm{HCV}$ is $~ 1$ to $3 \%$ over the course of 30 years. ${ }^{1} \mathrm{HBV}$ can integrate into the human genome and replicate within liver cells. This directly increases the carcinogenic activity in the liver through various signaling pathways. Unlike HBV, HCV is an RNA virus that cannot integrate into the human genome. Like HBV, HCV has a multifactorial role in the development of liver cancer including liver fibrosis, interaction with immune cells, affecting metabolic process, impacting apoptosis and cellular survival.

The Indian enigma is evident in the paradox of 50 to $80 \%$ prevalence of $H$. pylori infection that does not corroborate with the incidence of gastric cancer. This could be due to the mildly pathogenic variants of bacteria (NAB47 and NAD1) among the Indian population and other factors (dietary, tobacco, and socioeconomic status) that may mask the oncogenic effect of this infection. $H$. pylori infection causes chronic inflammation and theoretically fits the role of a promoter in the multistage model of carcinogenesis. ${ }^{13}$ It induces cell proliferation that in turn increases the risk for error in DNA replication and predisposes mucosal cells for transformation by dietary or endogenous mutagens. ${ }^{13}$ The likelihood of $\mathrm{N}$-nitrosamine formation is enhanced as inflammatory cells increase the conversion of nitrates to nitrites. This possibly could be the reason for the epidemiologic observation in some population, where high dietary nitrates increase the risk of gastric cancer. ${ }^{13}$

Research into the etiology of sporadic colon cancer has focused on dietary influences. Trend analysis reveals that fats are consistent risk factors for colon cancer and fiber is consistently protective. ${ }^{13}$ Antibacterial strategies as in the case of $H$. pylori model are unlikely to play a role in the prevention of colon cancer. Many species of bacteria exist in the gut, and there is a possibility that specific bacterial species play direct role in colon carcinogenesis. However, as there is no specific organism that is the culprit of colon cancer, prevention strategies should focus on diet as it influences bacterial pathogenesis. The developing world also harbors parasites associated with human cancer such as Schistosomiasis for bladder cancer and liver flukes for cholangiocarcinoma (cancer of bile duct).

Globally, HPV is the most common sexually transmitted virus. During the period 1955 to 1992, there was a decline of $\sim 70 \%$ mortality due to cervical cancer among countries with preventive health services. Safe sexual practices could prevent majority of these infections (HPV, hepatitis and HIV). Universal guidelines for screening and transfusion of blood products and the disposal of needles/syringes will enable reducing the source of exposure. Vaccination strategies will address the risk of hepatitis and HPV infections.

Kaposi's sarcoma is the most common cancer in untreated HIV-positive individuals. Kaposi sarcoma associated herpes virus is known as herpesvirus-8. HTLV-1 is a retroviral infection that affects the T-cells, but rarely causes adult T-cell leukemia (2-5\% of infected individuals) or muscle disorders (0.25-2\%). Although the cancer-causing mechanisms are poorly understood, T-cells infected by HTLV-1 can undergo genetic and epigenetic alterations. The Merkel cell polyoma virus causes a rare skin cancer "Merkel cell carcinoma" (MCC).

Endemic BL that occurs in the malaria belt is an EBV-positive malignancy that occurs several years after the primary infection with EBV. ${ }^{2} \mathrm{BL}$ is a monoclonal lymphoma of B-cell origin, wherein B-lymphocytes proliferate and there is a reduction in virus-specific cytotoxic T-cells due to the association with malaria. This results in an increase in the EBV viral load, enhancing the risk of chromosomal c-myc translocation, which is characteristic of BL. ${ }^{2}$ EBV may serve as a polyclonal B-cell mitogen thus setting the stage for chromosomal mutations.

-Fig. 1 depicts the list of risk factors associated with cancer, which are discussed in this article.

\section{Air Pollution}

Outdoor air pollution might cause cancer at sites other than lung through metabolism of the inhaled carcinogens. This includes breast, prostate, and colorectal regions. Household air pollution can happen from burning of solid fuels, passive smoking, and inadequate ventilation systems. Occupational exposure can happen from diesel and gasoline exhaust, polyaromatic hydrocarbons, inhalable dusts (metals, silica), work in mining, foundries, truck transportation, carbon black production, and with asphalt. The impact

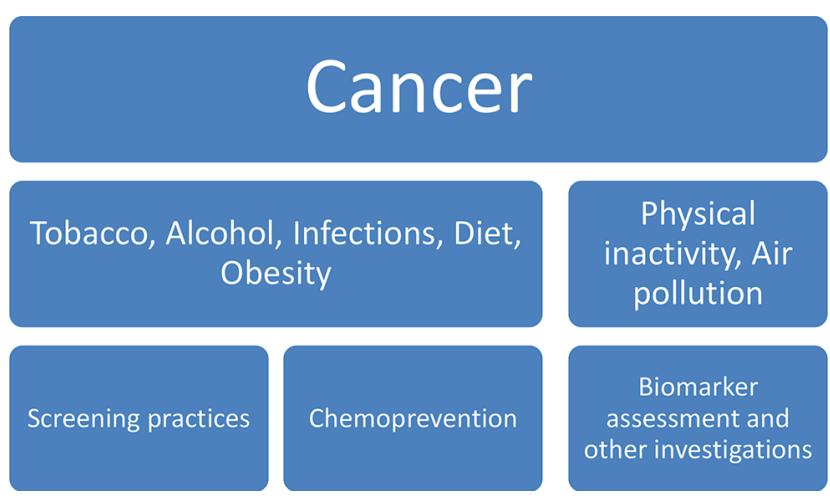

Fig. 1 Risk factors associated with cancer. 
includes disruption of several molecular process through direct or indirect (inflammation and oxidative stress) damage, inducing tumor suppressor gene inactivation and the activation of oncogenes, cell cycle alterations-dependent on TP53 activation, activation of energetic dysregulation, chromosome instability, inhibition of apoptosis, and induction of cell proliferation in somatic cells. ${ }^{14}$

IARC considers diesel exhaust as a probable human carcinogen. Other carcinogenic airborne pollutants derived from motor vehicle exhaust include benzene, polyaromatic hydrocarbons, and persistent organic pollutants (dioxin). A critical component of air pollution includes the airborne PM, of different sizes from various sources and human activities. In the respiratory tract, PM can cause inflammation, free radical formation, oxidative DNA damage, cytotoxicity, and mutagenesis. Household pollutants are generated from combustion of coal as well as due to sulfur dioxide and oxides of nitrogen.

Combustion of fossil and biomass fuels releases air pollutants such as gaseous pollutants (sulfur dioxide, nitrogen dioxide, carbon monoxide, volatile organic compounds, and PM including carbonaceous aerosol particles). Submicron $\mathrm{PM}_{2.5}$ is combustion related and of particular health concern as it contains numerous toxic compounds (acids and heavy metals), and tends to penetrate deeper into the lung than the larger PM generated by natural process such as soil particle mass.

A Chinese study ${ }^{15}$ found the risk among those burning solid fuel (coal, wood) to be four times more than those using clean energy. The usage of coal furnace for domestic heating carries twice the risk when compared with clean energy. Heatable brick bed exposure was associated with an eightfold increased risk. House layout and ventilation-related characteristics play important role in the risk of lung cancer. In the Indian context, since many females tend to work from within their homes and cook more frequently, the exposure at home has a stronger influence when compared with males.

It is imperative to monitor indoor exposure, regulate pollution levels and establish standard guidelines. It is a challenge to assess the exposure with regard to indoor air pollution. Using solid fuel for cooking and heating, and inadequate ventilation in the kitchen can significantly increase PM levels of all sizes during winter. Such association was not found during summers, ${ }^{15}$ as the ventilation-related characteristics of houses such as open windows enable reduction in levels of indoor air pollution.

\section{Physical Inactivity}

Physical inactivity, unhealthy diet, and obesity are associated with at least six cancers (colorectal, breast, stomach, liver, kidney, and endometrial). ${ }^{1}$ Physical inactivity by itself is attributable to $5 \%$ of cancer deaths. The Japanese public health center prospective study reports a fewer incidence of carcinoma stomach, colon, liver, and pancreas among individuals with daily total physical activity (PA). Judicious exercise tends to reduce fatigue, anxiety and improves self-esteem, strength, and cardiovascular fitness. Among cancer patients, low intensity PA can reduce the risk of complications and disease progression. Yoga triggers neurohormonal mechanism by suppression of sympathetic activity and improving autonomic function along with reducing anxiety, stress, insomnia, fatigue, and mood disturbances. Yoga could be helpful in managing psychological stress, thereby improving physical healing as both are closely related.

PA is not just a means of preventing cancer but is also a therapeutic strategy for delaying relapse and extending life expectancy after diagnosis of cancer. The proposed hypothesis for the physiological mechanism of health benefits rendered by exercise-centered PA includes regulation of sex hormones, insulin, and insulin-like growth factor, ${ }^{7}$ regulation of immunological function, and inhibition of free radical production. The "Inverted J hypothesis" discusses this issue and also deliberates the immune-suppressive effect of sedentariness and repeated exhaustive exercise, which increase the susceptibility to cancer. ${ }^{16}$ Long-term regular exercise provides protection against diseases associated with chronic inflammation, by reducing the body fat component and release of catecholamines, inhibiting the production of proinflammatory cytokines and promoting the release of anti-inflammatory cytokines by the muscles. ${ }^{16}$ Individuals on low-calorie diet are physically active and tend to have low serum levels of inflammatory cytokines such as tumor necrosis factor- $\alpha$, interleukin- 6 , and C-reactive protein.

Whether the nature of exercise is aerobic or anaerobic, natural killer (NK) cells are quickly mobilized to the peripheral circulation that in turn is linked to enhanced immune surveillance with transient increase in its cytotoxic activity. ${ }^{17}$ Evidence from studies suggests that mild physical exercise is safe and practicable during cancer therapy, and also improves quality of life during and posttreatment. We need to develop guidelines for planning the enhancement of PA and reducing sedentary behavior, together with details such as form, frequency, level, and duration of PA.

\section{Diet}

IARC reports on colon cancer indicate that a $17 \%$ increased risk exists for consumption of $100 \mathrm{~g} /$ day of red meat and $18 \%$ risk per $50 \mathrm{~g} /$ day of processed meat, and has identified them as potential carcinogens. The risk for cancer mortality was elevated with red meat (hazard ratio [HR]: 1.22 , 95\% confidence interval [CI]: 1.16-1.29) and processed meat (HR: 1.12, 95\% CI: 1.06-1.19). However, the consumption of red and processed meat is $<20 \%$ among the Indian population. ${ }^{1}$

A major component of the Indian diet comprises of vegetarian diet (primarily cereals and pulses), which has been associated with a lower risk of cancer when compared with the primarily nonvegetarian diet of the Western population. Turmeric (curcumin) has been found to significantly suppress cancer induction by dietary benzopyrene in animal studies. ${ }^{1}$

Among the Indian population, the following factors have been implicated in the causation of stomach cancer: spicy food, dried fish, and deep-fried cooking at high temperature (which generates polycyclic aromatic hydrocarbons). Many studies $^{4}$ demonstrate the inverse correlation between the incidence of lung cancer and diets high in fruit and vegetables. However, this needs to be validated through interventional 
studies that assess the correlation between dietary manipulation and reduction in the incidence of lung cancer. The macro- and micronutrients that comprise our diet may act as cancer inhibiting substances. Studies have shown that foods rich in $\beta$-carotene and lycopene carotenoids have an inverse relationship with cancer of the bladder, lung, and prostate. ${ }^{8}$ Evidence also suggests that dietary flavonoids (found in apples) and isothiocyanates (found in cruciferous vegetables) have an inverse relationship with the incidence of lung cancer. $^{8}$

Food is consumed as a part of the overall dietary pattern, wherein consumption includes a mixture of less healthful constituents as well as healthy dietary factors. There is clustering of lifestyle behaviors such as diet quality, PA, weight management, and smoking status. It is difficult to tease out the effect of a single element or behavior. The diet composition influences the microbiota of gut, which in turn affects the bioavailability of food components and metabolism of bioactive agents..$^{18}$ In recent times, the importance of microbiome in health and disease has gained recognition.

\section{Obesity: BMI ( $\left.\geq 30 \mathrm{~kg} / \mathrm{m}^{2}\right)$}

It accounts for $\sim 8 \%$ ( $10 \%$ in men and $6 \%$ in women) of all cancers. ${ }^{1}$ Singh et $\mathrm{al}^{19}$ report that among Indian women overweight (body mass index [BMI]: $25-29.9 \mathrm{~kg} / \mathrm{m}^{2}$ ) and obesity (BMI: $\geq 30 \mathrm{~kg} / \mathrm{m}^{2}$ ) have an OR of 1.06 and 2.27 , respectively, as compared with normal weight women for developing breast cancer.

The pathogenic link between obesity and susceptibility to cancer includes proposed novel candidate mechanisms such as chronic inflammation, oxidative stress, synergism between tumor cells and surrounding adipocytes, migrating adipose stromal cells, obesity-induced hypoxia, shared genetic susceptibility, and the functional defeat of immune function..$^{20}$ The main systems identified in this association include insulin, insulin-like growth factor-1, sex steroids, and adipokines. ${ }^{20}$ The worldwide rise in the incidence of obesity ${ }^{20}$ has been associated with the change in morphology of esophageal cancer from squamous to adenocarcinoma (AC). The Framingham Heart study ${ }^{20}$ shows that visceral adiposity, measured using multidetector computed tomography scan, is associated with cancer after adjustment for clinical risk factors and generalized adiposity. Certain conditions can foster particular tumors at specific sites due to the consequential effects of obesity, for example, heightened risk of cancer of the gall bladder when gall stones are present, and of esophageal $\mathrm{AC}$ as an effect of aggravated gastroesophageal reflux. Also, liver cancer can be a consequence of nonalcoholic steatohepatitis. ${ }^{20}$

Supraphysiological concentrations of insulin are known to have mitogenic effects through its anabolic and antiapoptotic effects, as insulin in normal levels does not induce somatic cell mutations. In obesity, insulin is highly concentrated and cancer cells have the ability to respond to its activation effect via the intracellular transduction pathways. ${ }^{20}$ Evidence suggests that energy accumulation leads to chronic inflammation in the body, and caloric restriction induces low inflammation.

\section{Screening and Preventive Strategies}

Available evidence suggests that during contemporary times, half of all cancers are preventable by practicing relevant measures. During the typical 10-minute encounter, the primary care physician should address the cancer-related risk factors and symptoms rather than focusing only on the patient's complaints. Otherwise, this practice increases the risk of delayed presentation as the patient fails to communicate the full breadth and complexity of their symptoms. This in turn results in missed opportunities for early detection of cancers.

Goldie et $\mathrm{al}^{21}$ report the cost-effectiveness of cervical cancer screening in five developing countries including India using computer-based models. Screening women once in their lifetime at the age of 35 years (either by visual inspection of cervix with acetic acid or DNA testing for HPV in cervical cell samples) could reduce the lifetime risk by 25 to $36 \%$, and would cost $<500$ USD per life year saved. Visual inspection for oral cancer screening by a trained health worker could lead to incremental (difference between intervention and control arms) cost per life year saved of USD 835 for all individuals and USD 156 for high-risk individuals.

Okonkwo et al $^{22}$ report that mammographic screening and clinical breast examination (every 5 years or biennially) among Indian women are predicted to reduce breast cancer mortality by 8 and $16 \%$, respectively. It is hypothesized that chest radiographic screening advances the diagnosis from clinical detection by $\sim 1$ year. $^{10}$

\section{Chemoprevention}

In chemoprevention, agents are used to prevent, inhibit, or reverse the process of carcinogenesis. The 5 -year overall survival for lung cancer patients is only $16 \%{ }^{4}$ and being the leading cause of cancer death in the world it makes an attractive case for chemoprevention. In SCLC and AC, the most common mutation is the loss of function of the p53 tumor-suppressor protein. However, restoring the function of p53 has not yet been translated therapeutically. Chemoprevention approaches will target processes such as interference with paracrine growth stimulation, suppression of inflammation, augmentation of apoptosis, improved immune surveillance, restoration of epithelial differentiation, and suppression of angiogenesis or invasion. ${ }^{4}$

Synthetic agents under investigation include nonsteroidal anti-inflammatory agents that are potent in vivo inhibitors of colon carcinogenesis, and difluoromethylornithine that is a polyamine synthase inhibitor known to have broad-spectrum in vitro and in vivo preventive activity. The role of the retinoid family as chemopreventive agent needs to be further investigated, with lung cancer being the prime target for these agents. The inverse relationship between the incidence of cancer and the selenium content of the soil (through locally grown foods) has been established. ${ }^{8}$ We need to gather evidence regarding whether supplementing the diet with these micronutrients is an effective method of cancer prevention.

\section{Biomarkers of Carcinogenesis}

Identification of novel cancer biomarkers is a challenge in cancer research, which could ideally be used as prognostic 
and diagnostic tools. Such biomarkers are present in tumor tissues or fluids, and include molecules such as DNA, mRNA, secreted proteins, transcription factors, cell surface receptors, and small metabolites. ${ }^{23}$

Ex-smokers can have improvement in bronchial metaplasia or dysplasia, but the risk of cancer remains increased. ${ }^{8}$ Another molecular marker in lung cancer (apart from p53 tumor suppressor gene) includes inactivation of the p16 tumor suppressor gene from either mutation or allelic loss or hypermethylation of $\mathrm{CpG}$ islands in its promoter region. Cyclo-oxygenase (COX-2) is an alternative form of COX and is expressed in response to growth factors. The expression of COX-2 has been proposed to be an early marker of a genetically altered epithelial cell, which could potentially become a cancer. Many COX-2 inhibitors have inhibitory effects on cell lines. ${ }^{8}$ In some studies, ${ }^{8}$ sputum sample examination for molecular markers shows sites of DNA loss among smokers. These losses are localized to chromosomal sites important in cancer.

EGFR is expressed in the normal epithelium of cells, but their increased expression is found in metaplastic lesions. Among lung cancer cases, it is found in $70 \%$ of squamous cell carcinoma and $50 \%$ in AC. Specific agents such as celecoxib for blocking COX-2 enzyme and ZD1839 for inhibition of EGF activated tyrosine kinase are currently being used for treatment, and have the potential for being used as preventive agents.

Other novel biomarkers that could be used include transcriptomic signature derived from endobronchial or nasal brushings, biopsy, or serum proteomics and analysis of volatile organic compounds in the exhaled breath. ${ }^{4}$

An ingenious method of delivering the chemopreventive agent includes the inhalational route, as it maximizes drug delivery to the target organ and minimizes the systemic effects. Individuals with premalignant lesions that are characterized by activated PI3K signaling could be targeted by using myoinositol. Different interventions could target phenotypes such as airway or parenchymal inflammation, tissue hypoxia, incipient angiogenesis, or excessive expression of growth factor. ${ }^{4}$

\section{Factors Which Prolong the Diagnosis of Lung Cancer}

Lung cancer is different from other smoking-related diseases as even former smokers are at significant risk subsequent to smoking cessation. Some studies ${ }^{4}$ report a favorable response to chemoprevention among former smokers when compared with the current smokers. Globally, SCLC was the most common histological type of nonsquamous cell lung cancer (NSCLC) before the year 1975 and post-1975 there is a shift toward AC. ${ }^{9}$ The increase in incidence of AC has been attributed to a change in smoking pattern (filter cigarettes with low tar and high nitrate content), as well as due to nonsmoking-related factors.

A high proportion of Asian women who were never-smokers were diagnosed with lung cancer when compared with women in the United States (>65 vs. $~ 10 \%) .{ }^{9}$ This could be due to the higher proportion of EGFR mutation in the Asian population. Such cancers among never-smokers are localized to the distal airways and favor the AC histology. ${ }^{9} \mathrm{Mu}$ et al' $^{7}$ study indicates that exposures other than smoking are the causal factors for female lung cancers. When compared with clean energy, the coal furnace used as a means of domestic heating in rural areas is associated with twice the risk of lung cancer. ${ }^{7}$

Vashistha et al's ${ }^{24}$ study reports a delay of $\sim 100$ days among Indian patients between developing symptoms and the definitive diagnosis. This duration extends by another 26 days for receiving the treatment. This confirms a study by Yurdakul et $\mathrm{al}^{2}$ in Turkey, where the average time for receiving treatment after developing symptoms was 130 days. Redaneil et $\mathrm{al}^{2}$ in their study at United Kingdom observed a median interval of $\sim 90$ days after developing symptoms for subsequent diagnosis with lung cancer. Radzikowska et $\mathrm{al}^{2}$ in their Polish study report a median duration of 90 days between onset of symptoms and administration of treatment. Also in Vashistha et al's ${ }^{24}$ study, chemotherapy was administered $\sim 2$ to 3 weeks following confirmed diagnoses. This extension of time may be due to the design of a multidisciplinary treatment plan, which could be hastened through greater collaboration among the healthcare staff. Evans et al's ${ }^{2}$ study in Australia observed a 30-day delay in initiation of chemotherapy and Shao et al's ${ }^{2}$ study in china observed a 2-month delay. However, these patients had undergone an initial surgical or radiotherapy intervention unlike in Vashistha et al's ${ }^{24}$ cohort.

Vashistha et al ${ }^{24}$ report patient and physician-level barriers for the mentioned time interval. At the patient level, barriers include lack of access to care and patients being ambivalent to their condition. Physician consultation involves out-of-pocket payment in India, and the level of poverty also contributes to the financial disadvantage. Physician-level delays are due to the differential tuberculosis (TB) diagnosis wherein the symptoms are mistaken to be similar. This is compounded by the initial radiographic signs that are quite similar. Data suggests that $25 \%$ of all new TB diagnosis is from India. ${ }^{5}$

Ramachandran et $\mathrm{al}^{25}$ during the course of their study in South India report that $<50 \%$ of physicians suspect lung cancer based on the presenting symptoms. The first differential diagnosis of TB was committed by $20 \%$ of the physicians. Physicians should exercise a high index of clinical suspicion among those at risk for lung cancer, and educate patients for accessing care at the onset of relevant symptoms.

Vashistha et $\mathrm{al}^{24}$ report that $~ 95 \%$ of patients with NSCLC present at their health center with either stage III or IV disease, while $~ 75 \%$ with SCLC present with extensive stage disease. This can be compared with data from United States ${ }^{2}$ that reports that $<70 \%$ patients with NSCLC or SCLC are diagnosed with either regionally invasive or distant metastatic disease upon presentation.

\section{Discussion}

In developing countries such as India, the role of cancer as a health problem gains precedence as its incidence increases directly with age, and advances in delivery of healthcare. Physician encounters could miss the opportunity to detect cancer at an early stage due to time pressures, either real or 
perceived, which might impede a thorough history taking or eliciting clinical signs. Other contributing factors that can impede diagnostic reasoning include language barriers, cognitive biases, and comorbidities.

\section{The Different Types of Cognitive Bias ${ }^{3}$}

Following are the three types of cognitive biases:

1. Anchoring bias: focusing exclusively on a single item of information.

2. Availability bias: over-reliance on already known or easily available information.

3. Commitment to a steer: initial diagnostic impressions.

These biases at the initial framing of the problem could lead to errors at the end of the diagnostic process, including misinterpretation of diagnostic test results. Also, the "epidemiological optimism" bias could adversely influence the diagnosis of cancer among low-risk patient groups such as young individuals and certain sociodemographic groups. Healthcare systems could fail to recognize and act on the abnormal tests related to cancer.

-Fig. 2 lists some of the focus areas in cancer prevention which are addressed in the Discussion section.

The age group at higher risk for cancer includes older patients who are multimorbid. Among such patients, symptoms compatible with known causes of chronic morbidity could be misinterpreted to reflect preexisting disease rather than a new problem. Patients may not adhere to the investigation plans due to emotional barriers such as fear of procedure-related pain/complications or adverse diagnosis.

Given the resource constraints in India, efforts should be made for cervical cancer screening at least once in the lifetime of a women aged $>30$ years. Oral cancer screening by trained healthcare workers is another cost-effective screening policy. This is relevant to the Indian context given the habits of tobacco, alcohol, and/or betel nut consumption. Vaccination for HPV (2 doses of quadrivalent vaccine given 6 months apart) among Indian girls could be a sustainable approach. ${ }^{12}$

A single polymorphism or dietary interaction may have synergistic effect on the overall risk of cancer. We may not be able to predict the type of cancer which an individual is susceptible toward, but risk assessment tools can enable focusing preventive activities for a particular cancer among the high-risk group. An important strategy is to develop accurate models for risk using both environmental and genetic (high-penetrance and low-penetrance alleles) factors. ${ }^{18}$

Molecular testing of the bronchial mucosa or epithelial cells for the presence of genetic or epigenetic changes could predict the risk of cancer. Chemoprevention agents could be trialed among such patients. Such markers may not be reliable predictors of cancer incidence, and an agent which improves the marker status may not decrease the incidence of cancer ultimately. Lung tumorigenesis could be better understood through advances in genomics, proteomics and molecular imaging studies, apart from the earlier practice of grading the histological changes. ${ }^{26}$

One of the main factors leading to lung cancer includes pulmonary inflammation. This could be the reason for chemopreventive efficacy of anti-inflammatory agents in murine models. ${ }^{4}$ The anti-diabetes agent Metformin is also used as a cancer chemopreventive agent. Some mechanisms for the same include decreased levels of insulin and insulin-like growth factor, and energy stress which leads to inhibition of liver kinase B1/AMP (adenosine

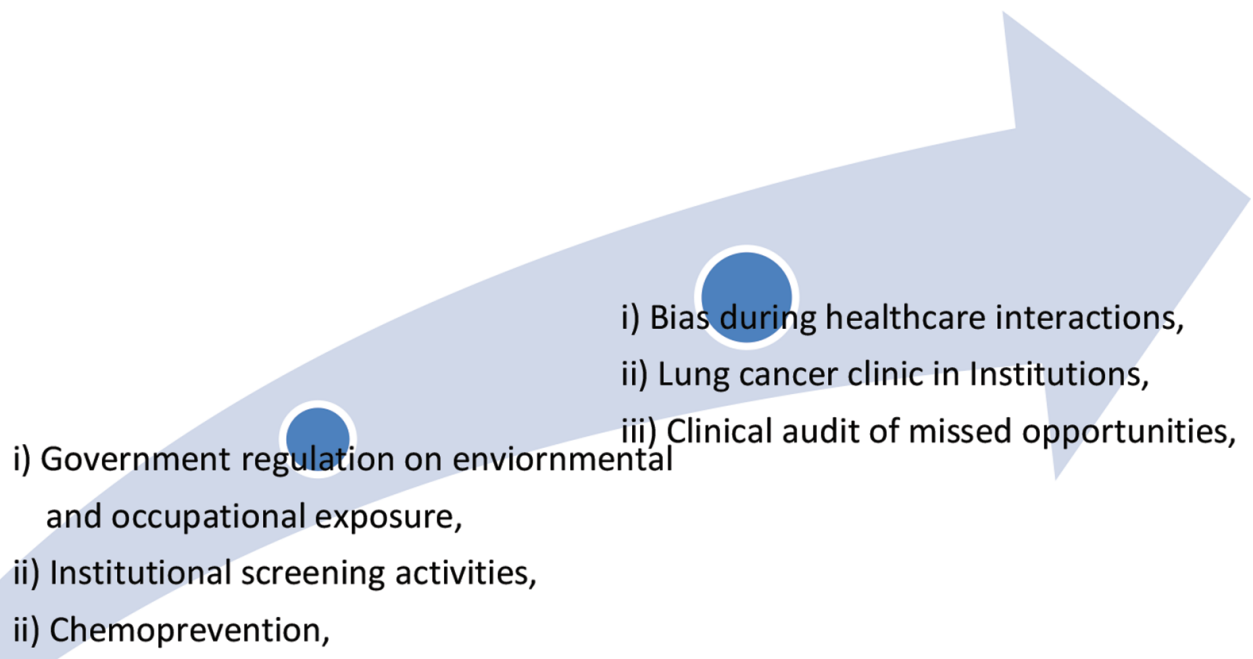

i) Gene polymorphism and dietary interaction,

ii) Molecular testing for genetic changes,

iii) Changes in Phenotype

iv) Non-smoke related risk factors for lung cancer,

Fig. 2 Focus areas in cancer prevention. 
cyclic monophosphate) activated protein kinase signaling. ${ }^{4}$ Prostacyclin analogues, specifically iloprost and thiazolidinediones such as Pioglitazone act through the same PPAR (peroxisome proliferator activated receptor gamma) receptor.

Although government regulations limit workplace and environmental exposure, further research in these areas will enable designing cost-effective lung cancer prevention strategies. Primary prevention efforts need to minimize the physical, chemical, and biological exposures to known carcinogens and for environmental interventions at work settings. The public and private healthcare centers should channelize efforts toward reducing diagnostic delays, which otherwise tend to unnecessarily postpone the diagnosis and management of lung cancer.

The role of estrogen in lung carcinogenesis needs to be further investigated. Lung cancer is being reported even among younger age groups with nontobacco etiology. Studies ${ }^{6}$ have found increased target mutations in such individuals when compared with smokers. Among nonsmokers, research should focus on environment and occupation-related risk factors attributable to lung cancer. Research could enable evidence-based preventive strategies at the biogenetic level for assessing the genetic and epigenetic interactions with environmental risk factors.

Population as well as institutional programs should screen for cancer of cervix, breast, and mouth, which could be diagnosed early and managed effectively when detected at initial stages. For cancers of the uterine corpus, female breast, testis, and melanoma, appropriate therapy yields a 5-year survival rate of $>75 \%$. However, for cancers of pancreas, liver, stomach, and lung, the survival is $<15 \% .^{5}$ Thus, a focus on treatment modalities for cancer management may not be an ideal strategy.

In India, there is a delay of 4 to 5 months for many patients from the time of diagnosis till they undergo treatment with chemotherapy. Developing a lung cancer clinic in hospital settings will enable the appropriate diagnosis and treatment of lung cancer. Supportive supervision of physicians will enable the practice of a high index of suspicion among at-risk individuals. The residual risk of developing lung cancer is significant among former smokers. Chemoprevention could be effective, particularly among former smokers, as they target premalignant lesions. Biomarkers that respond to these agents need to be validated. Although various leads are given through observational studies, no definitive preventive agent has been shown to be efficacious for lung cancer. Some of these agents include inhaled glucocorticoids, myoinositol, prostacyclin analogues, and thiazolidinediones. At present, smoking cessation seems to be the only preventive intervention for reducing the risk of lung cancer.

Chemoprevention agents should ideally target phenotypes expressing carcinogenic influences such as inflammation, angiogenesis, hypoxia, and epithelial differentiation, then to specific driver mutations. A definitive challenge in chemoprevention is to target the specific mutations of the molecule. Another advancing domain in the field of preventing lung cancer includes validating biomarkers of risk and response. Chemoprevention should be directed based on specific signatures (gene expression, proteomics, etc.) present in biopsy specimens from high-risk individuals. ${ }^{7}$

Clinical audit activity and case reviews need to be triggered by the presence of surrogate markers of missed opportunities. Such markers could include unusual patterns of multiple consultations (return visits), symptoms, or abnormal test findings suggesting the need for diagnostic evaluation of cancer and emergency presentations. Physicians should also take lead in retrospective reviews of randomly selected cancer cases over a defined period. However, audit of patient records includes potential for hindsight bias, missing documentation, and time needed for review process.

\section{Conclusion}

Identification of infectious etiology for specific cancers provides opportunities for preventing cancers by controlling the infections. This involves implementing public health measures or changes in cultural practices. As new infectious causes of malignancy are discovered, it is imperative to dissect the complex interplay between man and microbial flora to understand the pathogenesis of many malignancies. We need to follow screening guidelines and vaccination strategies for preventing cancers.

Consumption of deep fried and spicy food in the Indian diet contributes to the increasing burden of obesity, coupled with propensity of our community toward physical inactivity. Prevention efforts should include awareness and supportive initiatives for adopting a healthy lifestyle, which in turn can reduce the community's exposure to behavioral and dietary cancer risk factors. These should focus on quitting smoking, maintaining a healthy BMI, reducing alcohol consumption, exercise promotion, and consuming a healthy diet rich in fruits and vegetables. Further gains are possible by minimizing exposure to radiation (both natural and man-made), pollutants, and occupational chemicals, and vaccination against HPV and HBV.

The diagnosis of cancer involves interaction among multiple human and system components. Multidisciplinary approaches to prevent missed opportunities for cancer diagnosis should account for concepts such as shared mental models, optimal use of technology, and distributed cognition. Public health measures that are devised to tackle the burden of NCDs should adopt a consolidated approach for eliminating the shared environmental factors and social determinants driving this epidemic. Government interventions need to be supplemented by collaborative initiatives with the civil society and nongovernmental organizations.

\section{Conflict of Interest}

None declared.

\section{Reference}

1 Gandhi AK, Kumar P, Bhandari M, Devnani B, Rath GK. Burden of preventable cancers in India: time to strike the cancer epidemic. J Egypt Natl Canc Inst 2017;29(1):11-18 
2 Howley PM. Infectious disease causes of cancer: opportunities for prevention and treatment. Gordon Wilson lecture. Trans Am Clin Climatol Assoc 2015;126:117-132

3 Lyratzopoulos G, Vedsted P, Singh H. Understanding missed opportunities for more timely diagnosis of cancer in symptomatic patients after presentation. Br J Cancer 2015;112(Suppl 1): S84-S91

4 Nair M.K, Varghese C., Swaminathan R. Cancer: Current scenario, intervention strategies and projections for 2015. NCMH background papers - Burden of Disease in India. 2005;219-225

5 Shankar A, Dubey A, Saini D, et al. Environmental and occupational determinants of lung cancer. Transl Lung Cancer Res 2019;8(Suppl 1) :S31-S49

6 Goodman GE. Lung cancer. 1: prevention of lung cancer. Thorax 2002;57(11):994-999

7 Mu L, Liu L, Niu R, et al. Indoor air pollution and risk of lung cancer among Chinese female non-smokers. Cancer Causes Control 2013;24(3):439-450

8 Keith RL, Miller YE. Lung cancer chemoprevention: current status and future prospects. Nat Rev Clin Oncol 2013;10(6):334-343

9 Noronha V, Dikshit $\mathrm{R}$, Raut $\mathrm{N}$, et al. Epidemiology of lung cancer in India: focus on the differences between non-smokers and smokers: a single-centre experience. Indian J Cancer 2012;49(1):74-81

10 Mulshine JL, Smith RA. Lung cancer. 2: screening and early diagnosis of lung cancer. Thorax 2002;57(12):1071-1078

11 Rehm J, Shield KD. Alcohol use and cancer in the European Union. Eur Addict Res 2021;27(1):1-8

12 Sankaranarayanan R, Prabhu PR, Pawlita M, et al. Indian HPV Vaccine Study Group. Immunogenicity and HPV infection after one, two, and three doses of quadrivalent HPV vaccine in girls in India: a multicentre prospective cohort study. Lancet Oncol 2016;17(1):67-77

13 LoConte NK, Brewster AM, Kaur JS, Merrill JK, Alberg AJ. Alcohol and cancer: a statement of the American Society of Clinical Oncology. J Clin Oncol 2018;36(1):83-93
14 Parsonnet J. Bacterial infection as a cause of cancer. Environ Health Perspect 1995;103(8, Suppl 8) :263-268

15 Turner MC, Andersen ZJ, Baccarelli A, et al. Outdoor air pollution and cancer: an overview of the current evidence and public health recommendations. CA Cancer J Clin 2020;0:1-20

16 Yamaga T, Yamamoto S, Keiji M. The impact of physical activity on cancer prevention and survivorship. Phys Med Rehabil Res 2017;2(2):1-5

17 Kokila G, Smitha T. Cancer and physical activity. J Oral Maxillofac Pathol 2017;21(1):4-7

18 Meyskens FL Jr, Mukhtar H, Rock CL, et al. Cancer prevention: obstacles, challenges and the road ahead. J Natl Cancer Inst 2015;108(2):1-8

19 Singh P, Kapil U, Shukla N, Deo S, Dwivedi S. Association of overweight and obesity with breast cancer in India. Indian J Community Med 2011;36(4):259-262

20 De Pergola G, Silvestris F. Obesity as a major risk factor for cancer. J Obes 2013;2013:29154610.1155/2013/291546

21 Goldie SJ, Gaffikin L, Goldhaber-Fiebert JD, et al. Alliance for Cervical Cancer Prevention Cost Working Group. Cost-effectiveness of cervical-cancer screening in five developing countries. N Engl J Med 2005;353(20):2158-2168

22 Okonkwo QL, Draisma G, der Kinderen A, Brown ML, de Koning HJ. Breast cancer screening policies in developing countries: a cost-effectiveness analysis for India. J Natl Cancer Inst 2008;100(18):1290-1300

23 Abudawood M. Diabetes and cancer: a comprehensive review. J Res Med Sci 2019;24:94

24 Vashistha V, Choudhari C, Garg A, et al. The time required to diagnose and treat lung cancer in Delhi, India: an updated experience of a public referral center. Applied Cancer Research 2019;39:11

25 Ramachandran K, Thankagunam B, Karuppusami R, Christopher DJ. Physician related delays in the diagnosis of lung cancer in India. J Clin Diagn Res 2016;10(11):OC05-OC08

26 Keith RL. Lung cancer chemoprevention. Proc Am Thorac Soc 2012;9(2):52-56 